\title{
Ultrasound guided percutaneous thrombin injection of iatrogenic femoral artery pseudoaneurysms after coronary angiography and intervention
}

\author{
J D Ferguson, P J Whatling, V Martin, J Walton, A P Banning
}

\begin{abstract}
Ultrasound guided percutaneous thrombin injection has recently been described for the treatment of iatrogenic femoral pseudoaneurysms. Patient selection and technical aspects of this technique are still evolving and safety data, particularly after coronary intervention, remains limited. The percutaneous thrombin injection of femoral artery pseudoaneurysms in 13 consecutive patients, most of whom were receiving antiplatelet/anticoagulant treatment (aspirin 11, heparin 4, clopidogrel 6), is reported. Thrombin $(1000 \mathrm{U} / \mathrm{ml})$ was injected over several seconds until Doppler colour flow within the cavity ceased. The median dose of thrombin injected was $800 \mathrm{U}$ (range 200-1000 U) and the treatment was successful in all cases without complication. In one case, thrombus was visualised within the arterial lumen immediately after thrombin injection, but this dissolved spontaneously within five minutes without evidence of embolisation. In contrast to ultrasound guided compression, percutaneous thrombin injection of femoral pseudoaneurysms is a rapid, well tolerated, and successful technique even in patients receiving antiplatelet/anticoagulant treatment.

(Heart 2001;85:e5)
\end{abstract}

Keywords: ultrasound guided percutaneous thrombin injection; iatrogenic femoral artery pseudoaneurysm

Iatrogenic femoral artery pseudoaneurysms occur following $0.2-0.5 \%$ of diagnostic angiography cases and in up to $8 \%$ of coronary interventions. ${ }^{1}$ Risk factors include obesity, advanced age, aberrant femoral vascular anatomy, and concurrent antiplatelet/anticoagulant treatment. Pseudoaneurysms cause patient discomfort and frequently delay mobilisation and hospital discharge. Rarely, serious sequelae such as secondary infection or rupture may occur.

Apart from small pseudoaneurysms which may thrombose spontaneously, a definitive treatment is required in most cases. Ultrasound guided compression (UGC) ${ }^{2}$ remains the treatment of first choice in most centres and is successful in up to $90 \%$ of patients not receiving anticoagulation. ${ }^{3}$ Successful outcomes frequently depend on prolonged compression, with mean compression times of 30-104 minutes. ${ }^{4}$ Despite analgesia and sedation, UGC may cause intolerable discomfort and initial compression may fail requiring repeated attempts. Even after successful obliteration, recurrence of the pseudoaneurysms can occur in $20 \%$ of cases, ${ }^{5}$ particularly in those receiving anticoagulation. ${ }^{34}$ Until recently failed UGC has been managed by surgical repair which, although highly effective,
Department of Cardiology, John Radcliffe Hospital, Headley Way, Oxford OX3 9DU, UK

J D Ferguson

A P Banning

Department Vascular Surgery, John Radcliffe Hospital

P J Whatling

V Martin

J Walton

Correspondence to:

Dr Ferguson

Accepted 5 December 2000
Table 1 Pseudoaneurysm characteristics and dimensions

\begin{tabular}{|c|c|c|c|c|c|c|c|}
\hline Patient & Age: sex & Procedure & Anticoagulation & Cavity size (mm) & $\begin{array}{l}\text { Neck size } \\
\text { (mm) }\end{array}$ & $\begin{array}{l}\text { Cavity } \\
\text { volume } \\
(\mathrm{ml})\end{array}$ & $\begin{array}{l}\text { Total } \\
\text { thrombin dose } \\
(U)\end{array}$ \\
\hline 1 & $23: F$ & Renal angiogram & None & $33 \times 25 \times 39$ & $9 \times 3$ & 32.2 & 1000 \\
\hline 2 & $57: M$ & PTCA & $\mathrm{A}, \mathrm{C}$ & $13 \times 36 \times 11$ & $16 \times 5$ & 5.2 & 800 \\
\hline 3 & $58: M$ & PTCA+stent+IABP & $\mathrm{A}, \mathrm{C}$ & $55 \times 23 \times 24$ & $23 \times 4$ & 30.4 & 500 \\
\hline 4 & $77: M$ & Coronary angiography & $\mathrm{H}$ & $34 \times 25 \times 36$ & $22 \times 2$ & 30.6 & 1000 \\
\hline 5 & $72: \mathrm{F}$ & PTCA & $\mathrm{A}, \mathrm{C}$ & $48 \times 35 \times 20$ & $15 \times 3$ & 33.6 & 800 \\
\hline 6 & $61: M$ & PTCA & $\mathrm{A}, \mathrm{H}$ & $20 \times 10 \times 10$ & $22 \times 3$ & 2.0 & 600 \\
\hline 7 & 48:M & PTCA+stent & A,C & $30 \times 20 \times 15$ & $17 \times 2$ & 9.0 & 600 \\
\hline 8 & $70: F$ & PTCA+stent & $\mathrm{A}, \mathrm{H}, \mathrm{C}$ & $25 \times 50 \times 12$ & $20 \times 5$ & 15.0 & 600 \\
\hline 9 & $73: \mathrm{F}$ & Coronary angiography & A & $16 \times 16 \times 50$ & $20 \times 3$ & 12.8 & 500 \\
\hline 10 & 59:F & IABP & A & $52 \times 52 \times 32$ & $9 \times 4$ & 86.5 & 1000 \\
\hline 11 & $58: \mathrm{F}$ & PTCA+stent & $\mathrm{A}, \mathrm{C}$ & $50 \times 30 \times 20$ & $15 \times 3$ & 30.0 & 200 \\
\hline ^ 12 & 58:M & Coronary angiography & $\mathrm{A}, \mathrm{H}$ & $20 \times 20 \times 16$ & $5 \times 2$ & 6.4 & 800 \\
\hline 13 & $57: M$ & Coronary angiography & A & $24 \times 20 \times 15$ & $20 \times 4$ & 7.2 & 800 \\
\hline
\end{tabular}

Thrombin injection successfully thrombosed all pseudoaneurysms without complication apart from patient $12^{\star}$ in whom arterial thrombus developed immediately after the injection. The thrombus dissolved spontaneously without any vascular intervention. PTCA, percutaneous transluminal coronary angioplasty; IABP, intra-aortic balloon pump; A, aspirin; C, clopidogrel; H, heparin. 

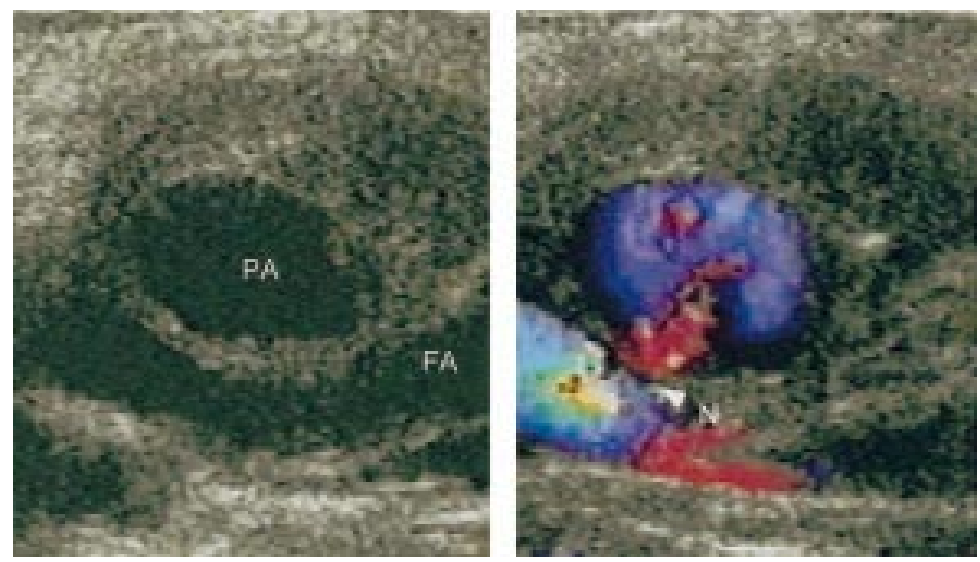

Figure 1 Pre-thrombin injection. (Left) Pseudoaneurysm cavity measuring $20 \times 16 \mathrm{~mm}$ with a patent femoral artery (FA). (Right) Colour Doppler flow within the arterial lumen and pseudoaneurysm cavity with accelerated flow through the short neck $(N)$ which measured $5 \times 2 \mathrm{~mm}$.
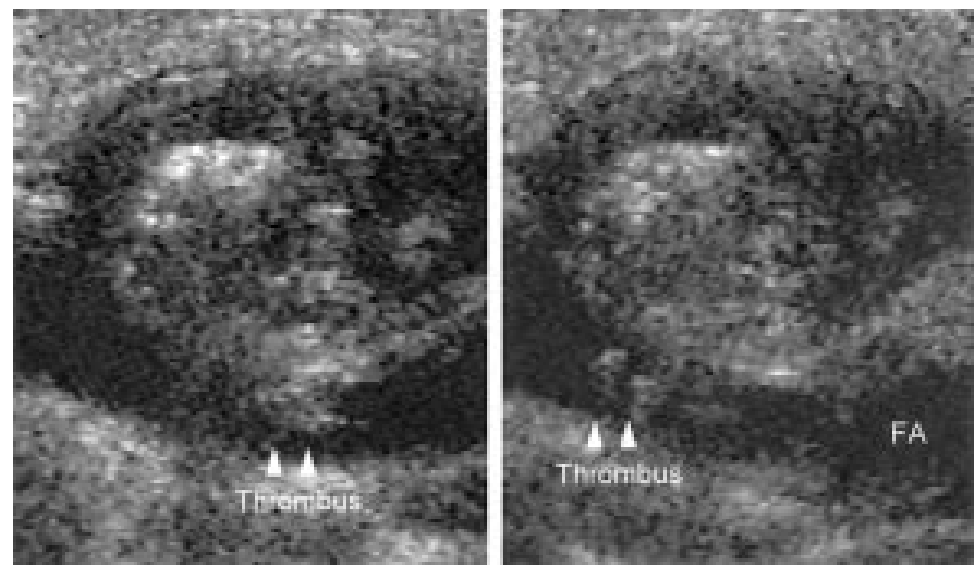

Figure 2 Post-thrombin injection. Echogenic thrombus within the pseudoaneurysm cavity and mobile thrombus within the arterial lumen attached to the neck of the pseudoaneurysm. Note the movement of the thrombus within the artery in systole and diastole.

does carry a significant incidence of postoperative bleeding and neuralgia. ${ }^{6}$ Serious anaesthetic complications during femoral artery repair are reported in up to $3 \%$ of cases. ${ }^{6}$

Liau and colleagues described ultrasound guided percutaneous injection of thrombin into the pseudoaneurysm in $1997 .^{7}$ This results in rapid thrombosis of the pseudoaneurysm cavity, demonstrated by complete obliteration of Doppler colour flow within seconds of the injection. Success rates of $93-100 \%$ have been reported, with no late recurrence seen on repeat ultrasound. ${ }^{14-9}$ The procedure is well tolerated by most patients without analgesia or sedation, and it can be used both in outpatient settings and in anticoagulated patients. ${ }^{49}$

This rapid and definitive treatment with relatively little patient discomfort is certainly appealing, providing it is safe and has good long term results. Like any new technique, the patient selection and technical aspects of thrombin injection are still evolving and safety data remain limited. We report on our experience of percutaneous thrombin injection of pseudoaneurysms which has raised important procedural safety issues.

\section{Patients}

Patients presenting with pseudoaneurysms between April 1999 and February 2000 who failed to respond to initial UGC were offered thrombin injection. Patients with evidence of peripheral vascular disease, congestive cardiac failure or previous thrombin exposure were excluded from the study. Written informed consent was obtained in all cases. Thirteen patients (seven male, median age 58 (range 23-77) years) underwent thrombin injection during the study period (table 1). Femoral artery cannulation had been performed for diagnostic coronary angiography in four patients, coronary intervention in seven patients (percutaneous transluminal coronary angioplasty (PTCA) alone in one patient, PTCA plus stent in six patients), renal angiography in one patient, and postoperative intra-aortic balloon pump in one patient.

\section{Methods}

The ultrasound examination was performed using an ATL, Ultramark 9 (Advanced Technology Laboratories, High Definition Image, Bothell, Washington, USA) with a $7-4 \mathrm{MHz}$ linear array transducer. Length, anteroposterior and transverse measurements of the pseudoaneurysm, and length and breadth of the neck of the cavity were recorded. Doppler waveforms of the common and superficial femoral arteries were recorded before and after injection. The skin adjacent to the transducer was prepared using alcohol swabs. Bovine thrombin (GenTrac Inc, Middleton, Wiscon$\sin$, USA) $1000 \mathrm{U} / \mathrm{ml}$ was drawn into a $2 \mathrm{ml}$ syringe. A 23 gauge needle was then inserted into the pseudoaneurysm so that the needle tip could be visualised within the cavity (close to the top, directed away from the neck) using two dimensional ultrasound. The thrombin was then manually injected over several seconds until the Doppler colour flow within the cavity had been obliterated or to a maximum dose of $1.5 \mathrm{ml}(1500 \mathrm{U})$. The total dose injected was recorded. Peripheral pulses and Doppler waveforms were then reassessed to confirm arterial patency. Patients were kept supine for one hour after the procedure. Repeat ultrasound examination was performed five days later.

\section{Results}

The median time to treatment was 10 (1-24) days after the initial procedure. Median cavity size was $15(2-86) \mathrm{ml}$, and cavity neck size ranged from $5-23 \mathrm{~mm}$ in length and $2-5 \mathrm{~mm}$ in breadth. Apart from one patient, all were on either antiplatelet agents or anticoagulants at the time of injection (aspirin 11, heparin 4, clopidogrel 6). The median dose of thrombin injected was $800 \mathrm{U}$ (range 200-1000 U) and the procedure was successful in all patients. Follow up ultrasound after five days showed complete cavity obliteration in all cases, and in $12 / 13$ patients there were no complications.

In one patient thrombus developed within the femoral artery lumen immediately after thrombin injection. The volume of the cavity was $6.4 \mathrm{ml}$ and $0.8 \mathrm{ml}(800 \mathrm{U})$ of thrombin had been injected. The thrombus measured $9 \times 4 \mathrm{~mm}$, was anchored to the neck of the pseudoaneurysm, and moved freely within the artery with phasic blood flow (figs 1 and 2). 
Although the normal Doppler triphasic waveform was distorted, the thrombus did not completely obstruct arterial flow, the distal pulses remained intact, and the patient experienced no symptoms. During continuous ultrasound scanning, the thrombus was seen to dissolve completely over five minutes without evidence of peripheral embolisation. Repeated scanning 24 and 96 hours later confirmed complete resolution of the thrombus.

\section{Discussion}

Percutaneous thrombin injection was successful in all cases in this series, most of whom were receiving antiplatelet treatment at the time of injection. Thrombosis was rapid $(<20$ seconds in all cases) and the procedure was well tolerated by all patients without sedation or analgesia. Seven out of 13 of these patients underwent treatment shortly after coronary intervention, but no ischaemic events were associated with thrombin injection.

In the six reported series including our own, 94 pseudoaneurysms have been treated by percutaneous thrombin injection. ${ }^{14-9}$ Both common and superficial femoral artery pseudoaneurysms and those with arteriovenous communication have been treated with percutaneous thrombin. At least 34 of the patients were treated shortly after coronary intervention, many were receiving antiplatelet agents (aspirin, ticlopidine or clopidogrel), and some were receiving heparin at the time of injection. ${ }^{4}$ The technique was successful in $97 \%$ of cases and, in the three patients in whom it failed, one had successful ultrasound guided compression and the other two required surgical repair. All series have reported good patient tolerance.

Careful attention to patient selection and thrombin injection technique is necessary to prevent complications. Most centres avoid this technique in patients with severe peripheral vascular disease or with previous exposure to thrombin products which may predispose to immune reactions. Cavity size and neck length should be assessed by ultrasound examination before thrombin injection. Care should be taken with small pseudoaneurysms $(<15 \mathrm{~mm}$ in diameter) to prevent relatively large volumes of thrombin being injected too quickly, thus spilling into the artery and causing luminal thrombus formation. The neck of the pseudoaneurysm, being formed by a needle or catheter, is usually only a few millimetres in width and has varied from $0-20 \mathrm{~mm}$ in length. A long narrow neck theoretically reduces the risk of thrombin spilling into the arterial circulation and, conversely, a broad short neck may expose the circulation to injected thrombin. ${ }^{10}$ In all cases, the key to preventing thrombin spilling into the artery is slow and cautious injection while observing Doppler flow within the cavity. Some authors have recommended prolonged balloon inflation over the neck of the aneurysm to protect the arterial lumen, ${ }^{11}$ but the need for further arterial puncture and the potential for stasis of arterial blood distal to the balloon has made this technique unattractive in most cases. ${ }^{12}$
With only limited safety data, thrombin is currently not licensed for the treatment of pseudoaneurysms, and patients receiving thrombin injection should do so on a named patient basis only. In previous studies, bovine thrombin was used but human thrombin is increasingly available and should be used preferentially to reduce the risk of immune reactions that have rarely been reported after topical bovine thrombin treatment. ${ }^{13-15}$ Concentrations of thrombin reconstituted with normal saline have varied from $100 \mathrm{U} / \mathrm{ml}$ to $1000 \mathrm{U} / \mathrm{ml}$ with a total of between 100$2000 \mathrm{U}$ of thrombin being required for effective cavity thrombosis. More dilute thrombin facilitates accurate titration of the total thrombin dose but may require greater injectate volumes. This potentially increases the risk of thrombin spilling into the arterial lumen, especially when treating small cavities. It is our opinion that concentrations of between 500$1000 \mathrm{U} / \mathrm{ml}$ should suffice in most cases.

Luminal thrombus has been reported in three femoral artery pseudoaneurysms. ${ }^{10}$ In our case the thrombin had probably been injected too quickly, thereby spilling into the arterial lumen. The entire cavity had thrombosed within a second and thrombus had formed within the arterial lumen immediately after the injection. With only partial arterial obstruction, no immediate intervention was required and the thrombus resolved spontaneously during a short period of observation. The two other cases required urokinase infusions and successful thrombolysis resulted in good long term results. ${ }^{10}$ No other serious complications of treating femoral artery pseudoaneurysms have been reported. Luminal thrombus has also been reported in two paediatric brachial artery pseudoaneurysms, one requiring embolectomy and the other resolving with heparin infusion alone. ${ }^{10}$ It is likely that thrombin which inadvertently leaks into the circulation is neutralised by dilution and anticoagulant factors, such as thrombomodulin and antithrombin III. ${ }^{10}$ However, when relatively large volumes of thrombin leak into the artery, or when there is reduced arterial flow which may facilitate thrombosis, these endogenous anticoagulants will provide insufficient protection and thrombus may form. Both our case, and that of the child in which the thrombus resolved with heparin infusion alone, ${ }^{10}$ show that thrombus may dissolve spontaneously thus obviating the need for vascular intervention.

In conclusion, ultrasound guided percutaneous injection of thrombin is a rapid and effective treatment for most pseudoaneurysms, including patients receiving antiplatelet agents after coronary intervention. The risk of luminal thrombosis is low but appears to be greatest when treating small pseudoaneurysms with a short neck. If these pseudoaneurysms do not thrombose spontaneously, we would recommend UGC before considering thrombin injection. No irretrievable thrombotic or severe systemic complications have been reported and, compared to the alternatives of painful UGC or surgical repair with its attendant risks, the safety profile of carefully performed 
thrombin injection appears acceptable. We are increasingly advocating early definitive treatment of pseudoaneurysms using thrombin injection which should minimise patient discomfort and accelerate mobilisation.

1 Wixon CL, Philpott JM, Bogey WM, et al. Duplex-directed thrombin injection as a method to treat femoral artery pseudoaneurysms. F Am Coll Surg 1998;187:464-6.

2 Fellmeth BD, Roberts AC, Bookstein JJ, et al. Postangiographic femoral artery injuries: nonsurgical repair with ographic femoral artery injuries: nonsurgical repair

3 Dean SM, Olin JW, Piedmonte M, et al. Ultrasound-guided compression closure of postcatheterization pseudoaneurysms during concurrent anticoagulation: a review of twenty-seven patients. 7 Vasc Surg 1996;23:28-34.

Kang SS, Labropoulos N, Mansour MA, et al. Percutaneous ultrasound guided thrombin injection: a new method for treating postcatheterization femoral pseudoaneurysms. $\mathcal{F}$ Vasc Surg 1998;27:1032-8.

5 Dorfman GS, Cronan JJ. Postcatheterization femoral artery injuries: is there a role for nonsurgical treatment? [editorial comment]. Radiology 1991;178:629-30.

6 Lumsden AB, Miller JM, Kosinski AS, et al. A prospective evaluation of surgically treated groin complications following percutaneous cardiac procedures. Am Surg 1994;60:132-7.

7 Liau CS, Ho FM, Chen MF, et al. Treatment of iatrogenic femoral artery pseudoaneurysm with percutaneous thrombin injection. F Vasc Surg 1997;26:18-23.
8 Taylor BS, Rhee RY, Muluk S, et al. Thrombin injection versus compression of femoral artery pseudoaneurysms. $\mathcal{F}$ Vasc Surg 1999;30:1052-9.

9 Brophy DP, Sheiman RG, Amatulle P, et al. Iatrogenic femoral pseudoaneurysms: thrombin injection after failed US-guided compression. Radiology 2000;214:278-82.

10 Lennox A, Griffin M, Nicolaides A, et al. Regarding "percutaneous ultrasound guided thrombin injection: a new method for treating postcatheterization femoral pseudoaneurysms" [letter; comment]. F Vasc Surg 1998;28:1120-1.

11 Loose HW, Haslam PJ. The management of peripheral arterial aneurysms using percutaneous injection of fibrin adhesives. Br F Radiol 1998;71:1255-59.

12 Elford J, Burrel C, Roobottom C. Ultrasound guided percutaneous thrombin injection for the treatment of iatrogenic pseudoaneurysms. Heart 1999;82:526-7.

13 Muntean W, Zenz W, Edlinger G, et al. Severe bleeding due to factor $\mathrm{V}$ inhibitor after repeated operations using fibrin sealant containing bovine thrombin. Thromb Haemost 1997;77:1223

14 Chouhan VD, De la Cadena RA, Nagaswami C, et al. Simultaneous occurrence of human antibodies directed against fibrinogen, thrombin and factor $\mathrm{V}$ following the exposure to bovine thrombin: effects on blood coagulation, protein $\mathrm{C}$ activation and platelet function. Thromb Haemost 1997;77:343-9.

15 Carroll JF, Moskowitz KA, Edwards NM, et al. Immunological assessment of patients treated with bovine fibrinogen as a hemostatic agent. Thromb Haemost 1996;76:92531 . 